\title{
3rd Meeting of the European Society of Neurosonology and Cerebral Hemodynamics
}

\author{
Guest Editors \\ D. Grosset, Glasgow \\ D. Russell, Oslo
}




\section{KARGER}

\section{S. Karger}

Medical and Scientific Publishers Basel $\cdot$ Freiburg $\cdot$ Paris $\cdot$ London

New York $\cdot$ New Delhi $\cdot$ Bangkok Singapore $\cdot$ Tokyo $\cdot$ Sydney
Drug Dosage

The authors and the publisher have exerted every effort to ensure that drug selection and dosage set forth in this text are in accord with current recommendations and practice at the time of publication. However, in view of ongoing research, changes in government regulations, and the constant flow of informain government regulations, and the constant flow of informa-
tion relating to drug therapy and drug reactions, the reader is tion relating to drug therapy and drug reactions, the reader is
urged to check the package insert for each drug for any change
in indications and dosage and for added warnings and precauin indications and dosage and for added warnings and precau-
tions. This is particularly important when the recommended agent is a new and/or infrequently employed drug.
All rights reserved

No part of this publication may be translated into other languages, reproduced or utilized in any form or by any means, electronic or mechanical, including photocopying, recording, microcopying, or by any information storage and retrieval system, without permission in writing from the publisher or, in system, without permission in writing from the publisher or, in
the case of photocopying, direct payment of a specified fee to the Copyright Clearance Center (see 'General Information').

(C) Copyright 1998 by S. Karger AG,

P.O. Box, CH-4009 Basel (Switzerland)

Printed in Switzerland on acid-free paper by

Reinhardt Druck, Basel

ISBN 3-8055-6703-0 


\section{Cerebrovascular}

Diseases

\section{European Society of}

Neurosonology and

\section{Cerebral Hemodynamics}

Society President

Prof. David Russell

Society Officers

Dr. Rob Ackerstaff

Dr. Eva Bartels

Dr. Donald Grosset

Dr. Jürgen Klingelhöfer

Dr. Kurt Niederkorn

Prof. E. Bernd Ringelstein

Prof. Elietta M. Zanette

\section{Organising Committee}

\section{Local Organising Committee}

Chairman Dr. Donald Grosset

Local Committee

Mr. Iain Sim

Dr. Ian Piper

Prof. David Evans

\section{Abstract Review Committee}

Dr. R.G.A. Ackerstaff

Prof. D. Russell

Prof. E.B. Ringelstein

Prof. D.H. Evans

Dr. H. Markus

Prof. E.M. Zanette

Dr. D. Grosset
The Organising Committee is grateful to the participants who have agreed to help with the basic and advanced tutorials section, which remains an important part of the Neurosonology and Cerebral Hemodynamics meeting.

The following are confirmed tutors:

Dr. Hugh Markus, Dr. Dimitrios Georgiadis, Dr. Mario Siebler, Mr. Iain Sim, Dr. Sheila Byrd. 


\section{Scientific Program}

Monday, May 25, 1998

09.05

Welcome and Introduction

D. Grosset

\begin{tabular}{ll}
\hline & Emboli Studies: Session $\mathbf{1}$ \\
\hline 09.15 & Transcranial Doppler and Emboli
\end{tabular}

09.35-10.30 Oral Presentations (Abstracts 1-5)

Coffee Break

\begin{tabular}{|c|c|c|}
\hline & \multicolumn{2}{|l|}{ Coffee Break } \\
\hline & Emboli Studies: Session 2 & Chairman: D. Grosset \\
\hline 11.00 & Microemboli Signals in Patients with Cardioembolic Sources & Keynote Speaker: D. Georgiadis \\
\hline $11.20-12.10$ & Oral Presentations (Abstracts 6-10) & \\
\hline 12.10 & Poster Presentations (Abstracts 52-82) & \\
\hline \multirow[t]{2}{*}{$13.00-14.00$} & Lunch & \\
\hline & Power Doppler and Other Studies & Chairman: E.B. Ringelstein \\
\hline 14.00 & The Physics of Power Doppler & Keynote Speaker: D. Evans \\
\hline 14.15 & Diagnostic Studies Using Power Doppler Techniques & Keynote Speaker: J. Wardlaw \\
\hline \multirow[t]{3}{*}{$14.30-15.25$} & Oral Presentations (Abstracts 11-15) & \\
\hline & Coffee Break & \\
\hline & Monitoring of Brain Function & Chairman: K. Neiderkorn \\
\hline$\overline{16.00}$ & $\begin{array}{l}\text { Multimodality Recording of Brain Function and Structure in } \\
\text { Acute Stroke }\end{array}$ & Keynote Speaker: M. Siebler \\
\hline 16.20 & Functional MRI and the Occipital Cortex & Keynote Speaker: B. Condon \\
\hline $16.40-17.50$ & Oral Presentations (Abstracts 16-22) & \\
\hline 19.00 & Civic Reception & \\
\hline
\end{tabular}




\begin{tabular}{|c|c|c|}
\hline \multicolumn{3}{|c|}{ Tuesday, May 26, 1998} \\
\hline & Cerebral Blood Flow Studies & Chairman: J. Klingelhöfer \\
\hline 09.05 & Cerebral Blood Flow Studies: Acute Stroke & Keynote Speaker: K.W.M. Muir \\
\hline \multirow[t]{3}{*}{$09.25-10.35$} & Oral Presentations (Abstracts 23-29) & \\
\hline & Coffee Break & \\
\hline & Carotid Artery Disease & Chairman: D. Evans \\
\hline $11.05-12.05$ & Oral Presentations (Abstracts 30-35) & \\
\hline 12.05 & Atheroma Detection in Carotid Stenosis & Keynote Speaker: H. Sillesen \\
\hline 12.25 & Doppler Studies in Carotid Endarterectomy & Keynote Speaker: R. Naylor \\
\hline 12.45 & Business Meeting, Members of the ESNCH Society & \\
\hline \multirow[t]{2}{*}{$13.00-14.00$} & Lunch & \\
\hline & Contrast Studies and Colour Coding & Chairpersons: D. Russell, E. Bartels \\
\hline 14.00 & $\begin{array}{l}\text { Echo Contrast Agents in Transcranial Colour-Coded Sonography } \\
\text { of the Vertebrobasilar System }\end{array}$ & Keynote Speaker: E. Bartels \\
\hline \multirow[t]{3}{*}{$14.15-15.40$} & Oral Presentations (Abstracts 36, 47, 38-44) & \\
\hline & Coffee Break & \\
\hline & Analysis Studies and TCD Waveforms & Chairman: R. Ackerstaff \\
\hline 16.10 & Automated Analysis of Event-Related Cerebral Blood Flow & Keynote Speaker: M. Deppe \\
\hline $16.30-17.30$ & Oral Presentations (Abstracts 45, 46, 37, 48-51) & \\
\hline 17.45 & Introduction to the 7th European Stroke Conference & M. Hennerici \\
\hline 18.00 & 4th ESNCH Meeting Announcement, Venice 1999 & E. Zanette \\
\hline 18.05 & Closing Remarks & D. Russell \\
\hline
\end{tabular}

$19.00 \quad$ Gala Dinner


This issue contains the abstracts (camera-readycopy) of the 3rd Meeting of the European Society of Neurosonology and Cerebral Hemodynamics, Glasgow, UK, May 24-26, 1998 and is not available online for technical reasons. 